\title{
LA FORMACIÓN DE INGENIEROS EN EL SIGLO XXI
}

Jorge Dettmer González

\section{RESUMEN}

Ante un entorno desbordante de cambios -tecnológicos, sociales, culturales, económicos...-, la educación superior ha necesitado responder de forma radical: sólo este ajuste le permitirá estar a la altura de la transformación que se le está exigiendo.

La enseñanza de la ingeniería representa un renglón enormemente sensible a estas nuevas y complejas realidades académicas, administrativas y curriculares que surgen de unos requerimientos sin precedente pero, sobre todo, de un país también en plena transición. Sólo al cuestionar a profundidad los fines últimos de su vocación, será que los ingenieros de este milenio redefinan su papel y contribución para la mejora y fortalecimiento de esta nueva sociedad que hoy empieza a desplegarse.

\section{INTRODUCCIÓN}

En las últimas dos décadas, la mayor parte de los sistemas de educación superior en general y de enseñanza de la ingeniería en particular, han experimentado importantes transformaciones con objeto de responder a las nuevas y complejas realidades derivadas de fenómenos tales como: la globalización; la explosión de nuevas tecnologías (particularmente de las tecnologías de la información y las telecomunicaciones); el surgimiento de nuevas formas de producción y utilización del conocimiento; así como la conformación de nuevas 
estructuras organizacionales de las empresas, basadas en sistemas de relaciones menos jerárquicas y más horizontales.

Para ajustarse a éstas y otras realidades económicas, tecnológicas y ocupacionales, las instituciones de educación superior -particularmente aquéllas abocadas a la enseñanza de la ingeniería-, se han visto precisadas a introducir importantes cambios en sus estructuras académicas, administrativas y curriculares a fin de elevar la calidad de la enseñanza y atender las demandas de la nueva organización industrial y los servicios. Puesto que reformas parecidas han tenido lugar en otras latitudes, algunos autores han visto en este fenómeno una suerte de convergencia de políticas, sistemas, instituciones, curricula y grados académicos, propiciados por los procesos de globalización e internacionalización de la educación superior, en tanto que otros explican el mismo fenómeno como consecuencia de la diferenciación institucional.

Mientras que -al menos teóricamente- la convergencia estimularía la homogenización y estandarización de los sistemas de enseñanza superior, la diferenciación promovería una mayor orientación de este tipo de instituciones hacia el mercado. En los hechos, sin embargo, ambas parecen formar parte de un continuum, observándose incluso convergencia o diferenciación según el nivel de análisis que se estudie (es decir, al nivel de las políticas, sistema de educación superior, instituciones concretas, curriculum, etcétera).

En México, los estudios sobre procesos de convergencia en la educación superior han sido casi inexistentes, predominando más bien los análisis en torno a la diferenciación y la segmentación institucional. No obstante, la profundización de la globalización y la internacionalización económica -especialmente a partir de la firma del Tratado de Libre Comercio de América del Norte (TLCAN)-, ha propiciado un mayor interés por la posible convergencia de los sistemas de educación superior de México y sus vecinos del norte. En este contexto, el objetivo central de este trabajo es, por un lado, analizar de qué manera la firma del TLCAN ha estado influyendo en la reforma de la 
enseñanza de la ingeniería en México y, por el otro, determinar hasta qué punto el actual proceso de formación de ingenieros puede ser entendido y explicado como resultado de los procesos de convergencia y/o diferenciación.

El trabajo que a continuación se presenta, se divide en seis partes. En la primera, se analizan los impactos sociales y ocupacionales provocados por las nuevas tecnologías; particularmente las tecnologías de la información y la comunicación. En la segunda, se revisan las experiencias de diferentes países, en los cuales la disponibilidad de fuerza de trabajo calificada -en especial de ingenieros-, ha estado asociada, significativamente, al desarrollo económico. En la tercera parte, se analiza el papel que tradicionalmente han desempeñado las universidades en la formación de recursos humanos de alto nivel, especialmente de ingenieros, así como el papel que les tocará jugar en el futuro inmediato. En el cuarto apartado, se examina el impacto del cambio tecnológico en el curriculo de ingeniería. En el quinto, se realiza una síntesis de la trayectoria de formación de ingenieros en México. En el sexto y último apartado, se plantean las perspectivas futuras de formación de ingenieros en México, en el marco del Tratado de Libre de Comercio de América del Norte (TLCAN), y de las tendencias hacia la convergencia y/o diferenciación en la educación en ingeniería.

\section{IMPACTOS SOCIALES Y OCUPACIONALES DE LAS NUEVAS TECNOLOGÍAS}

El punto de partida de este trabajo, denota que las aceleradas transformaciones científico-tecnológicas observadas a partir de la Segunda Mundial, han dado lugar al surgimiento de un nuevo paradigma tecnológico -identificado con el nombre de "Tercera Revolución Industrial»- que, entre otros efectos, está propiciando cambios significativos en la orientación y el contenido de la enseñanza de la ingeniería, así como en el desempeño ocupacional y el prestigio social de los ingenieros como grupo profesional. 
En contraste con la primera Revolución Industrial (sustentada en el creciente uso de la energía de vapor), y la segunda (basada en la producción de energía eléctrica y el petróleo baratos), dos parecen ser las características específicas de esta nueva revolución tecnológica: 1) se trata de un conjunto de descubrimientos y desarrollos tecnológicos que afectan tanto a los procesos de producción como a los productos; 2) la materia prima esencial de tales descubrimientos y desarrollos tecnológicos es la información. Por consiguiente, lo característico de las llamadas "nuevas tecnologías" (entre las que destacan la electrónica, informática y telecomunicaciones) es su interdependencia, ya que los avances de cada campo facilitan el progreso en otros campos, con base en la comunicabilidad de la información ${ }^{1}$.

El acelerado desarrollo, difusión y aplicación de estas nuevas tecnologías, ha modificado radicalmente la estructura económica y social tanto de los países altamente industrializados como de los atrasados.

En la esfera de la producción, dichas tecnologías han dado lugar, entre otros aspectos, al surgimiento de nuevas organizaciones productivas caracterizadas por: 1) asumir con rapidez el cambio tecnológico; 2) adoptar una estructura flexible (basada en una amplia redistribución de responsabilidades) que opere en mercados altamente segmentados y concentrados; 3) procurar la mejora continua y, 4) promover el entrenamiento múltiple, la recalificación y el aprendizaje permanente de su personal ${ }^{2}$.

\footnotetext{
${ }^{1}$ Dado que los flujos de información pueden ser tratados como estructuras binarias (mediante combinaciones infinitas de 1 y 0 ), existe la posibilidad de interconectar varios sistemas de información que operan sobre la base de una lógica común. Cfr. Castells, Manuel, Antonio Barrera, et.al., El desafio tecnológico en España. España y las nuevas tecnologías, Madrid, Alianza Editorial, 1986, p.24.

${ }^{2}$ Pérez, Carlota, "Nuevo patrón tecnológico y educación superior: una aproximación a la empresa", en varios autores, Reunión Internacional de Reflexión sobre Nuevos Roles de la Educación Superior a Nivel Mundial: el caso de América Latina y del Caribe, futuro y escenarios deseables. vol. 3, Retos Científicos y Tecnológicos. Caracas, UNESCO/CRESALC, 1991, pp.23-49.
} 
En el ámbito laboral, estas nuevas tecnologías han contribuido a elevar la productividad de los trabajadores, modifican el número de empleos disponibles en las distintas ramas y sectores económicos ${ }^{3}$ y transforman significativamente tanto las características de los puestos de trabajo como el tipo de conocimientos, habilidades y destrezas requeridos para desempeñarlos ${ }^{4}$.

Puesto que uno de los rasgos más significativos de las nuevas tecnologías es su alto contenido de conocimiento científico, ello ha conducido a revalorar el papel de los sistemas de educación superior y científico-tecnológicos, considerándolos como factores estratégicos para asegurar el aprendizaje tecnológico de cualquier nación 5 .

\section{DESARROLLO ECONÓMICO, EDUCACIÓN Y FORMACIÓN EN INGENIERÍA: ALGUNAS EXPERIENCIAS}

Un somero análisis del crecimiento económico alcanzado por diversos países desarrollados a partir de la Segunda Guerra Mundial, como Japón, por ejemplo, y otros de reciente industrialización (NIC's), muestra que dicho crecimiento estuvo acompañado de políticas tendientes a favorecer: 1) el

\footnotetext{
${ }^{3}$ Como resultado de estos cambios, desde hace varias décadas se viene observando, en casi todos los países, un acelerado crecimiento del empleo en el sector servicios en relación con aquéllos generados en la industria y la agricultura.

${ }^{4}$ En este sentido, C. Pérez destaca que parece haber una tendencia a reducir los requerimientos de calificaciones de nivel medio y a aumentar los de nivel inferior y superior, a la vez que se demandan especializaciones menos estrechas y más capacidades básicas "multipropósito" para el manejo de información. Al respecto véase Pérez, Carlota, "Las nuevas tecnologías: Una visión de conjunto", en Carlos Ominami (ed.), La tercera revolución industrial. Impactos internacionales del actual viraje tecnológico. Buenos Aires, Grupo Editor Latinoamericano, 1986, pp.53-54.

${ }^{5}$ Cimoli, Mario y Giovanni Dosi, "Tecnología y desarrollo. Algunas consideraciones sobre los recientes avances en la economía de la innovación", en Mikel Gómez, Miguel Sánchez y Enrique de la Puerta (comps.), El cambio tecnológico hacia el nuevo milenio, Barcelona, Ed. Icaria, 1992, p.41.
} 
sistema científico-tecnológico interno y, 2) las transformaciones organizacionales de sus sistemas educativos, particularmente, de sus centros de educación superior ${ }^{6}$.

Otros estudios destacan cómo, en el caso de los países menos industrializados, los ajustes introducidos en sus sistemas educativos, están aumentando sus capacidades científicotecnológicas nacionales ${ }^{7}$.

En el caso de América Latina, Teitel y Westphal ${ }^{8}$ señalan cómo la disponibilidad de ingenieros y científicos dotados de conocimientos técnicos adquiridos en las universidades y centros de investigación de la región, hizo posible la adquisición y recepción de tecnología en el sector manufacturero.

Si bien -como lo constata un estudio de J. J. Brunner ${ }^{-}$, entre 1965 y 1980, la cantidad de ingenieros y científicos dedicados a actividades de investigación y desarrollo en América Latina creció de manera significativa, su número continuó

\footnotetext{
${ }^{6}$ Acerca de este punto véanse: Gill-Chin Limm, "La transformación dinámica y el papel del gobierno: La lección de Corea y de los países de industrialización reciente"; Tun-Jen Chen, "Características distintivas del desarrollo económico de Taiwan, con referencia particular al papel de las medianas y pequeñas empresas"; Ruíz Durán, Clemente, "El bienestar social, el desarrollo económico y la organización empresarial". Todos estos trabajos se encuentran en: Martínez Legorreta, Omar (compilador), Industria, comercio y Estado. Algunas experiencias de la Cuenca del Pacífico, México, El Colegio de México. Véase también, Benachenhou, Abdellatyff, "Adquisición de conocimientos en los países subdesarrollados. Situación actual y perspectivas", en Foro Internacional, Vol.XXVIII, No.2, octubre-diciembre, 1987.

7 Para un análisis más detallado de este aspecto véase: Segal, Aaron, "De la transferencia de tecnología a la institucionalización de la ciencia y la tecnología", en Revista Comercio Exterior, Vol. 37, No.12, diciembre de 1987, pp.983-993. 8 Teitel, Simón y Westphal, Larry E., Cambio tecnológico y desarrollo industrial, México, Ed. Fondo de Cultura Económica, 1990, pp.58-59.

${ }^{9}$ De acuerdo con estimaciones de Brunner, mientras que en 1965 el número de ingenieros y científicos dedicados a actividades de Investigación y Desarrollo en América Latina no rebasaba los 15 000, para 1970 la cifra se había elevado a cerca de 38 000, llegando en 1980 a unos 91 000. Pese a este esfuerzo de formación de recursos humanos, la región latinoamericana participaba apenas con el $2.4 \%$ del total de ingenieros y científicos existentes a nivel mundial. Cfr. Brunner, José Joaquín. Recursos humanos para la investigación cientifica en América Latina, Santiago, FLACSO/IDRC, 1989, pp.32-33 y 288-289
} 
siendo muy reducido comparado con la proporción del mismo tipo de personal existente en los países desarrollados. Este hecho, aunado a la falta de consistencia entre las políticas educativas, de industrialización y de selección de tecnologías, entre otros factores, impidieron la consolidación de una capacidad científico-tecnológica autónoma en la región.

La fuerte crisis económica que atravesó América Latina durante la década de los ochenta, y que continuó durante los años noventa, contribuyó aún más a agravar esta situación produciendo un acelerado proceso de obsolescencia tecnológica manifestado en un serio deterioro de la calidad de los recursos humanos (formados por las universidades) y en el abandono de importantes líneas de investigación ${ }^{10}$.

En este contexto, ante el riesgo de permanecer como simples usuarios de las vertiginosas innovaciones científicas y tecnológicas que se están operando, algunos países y organismos ${ }^{11}$ de la región han planteado una nueva estrategia dirigida a reestructurar los sistemas educativos -en especial la enseñanza superior- y a superar los déficits de recursos humanos altamente calificados (principalmente en las áreas técnicas y científicas), a fin de lograr una auténtica competitividad y mayor equidad educativa, económica y social en la década de los noventa.

\footnotetext{
${ }^{10}$ Sagasti, R. Francisco, "Ciencia, tecnología y la encrucijada de América Latina", en Manuel Gamella y Mar Hernández de Felipe (coords.), Nuevas tecnologías y orden económico internacional, Ed. FUNDESCO, Madrid, 1990.

${ }^{11} \mathrm{Al}$ respecto véase: CEPAL. Transformación productiva con equidad. UNESCO, Santiago de Chile, marzo, 1990; CEPAL. Educación y conocimiento: Eje de la transformación productiva con equidad. UNESCO, Santiago de Chile, marzo, 1992.
} 


\section{UNIVERSIDAD Y FORMACIÓN PROFESIONAL EN AMÉRICA LATINA}

La mayoría de los autores ${ }^{12}$ que analizan el papel de la Universidad en la generación de una capacidad científicotecnológica autónoma han tendido a asignarle, como una de sus funciones primordiales, la formación de profesionales y científicos que participen en el proceso de desarrollo.

En la actualidad, el reto fundamental de las universidades de América Latina, y de México en particular, es la formación de profesionales que se vinculen a las tareas del desarrollo desde una ubicación altamente especializada.

De acuerdo con Brunner ${ }^{13}$, lo que se requiere, al pensar en un especialista en desarrollo, es una categoría completamente diferente del practicante. Se trata de hombres de acción, al estilo de los empresarios; de hombres de gestión, al estilo de los administradores; y de hombres de descubrimiento, al estilo de los científicos. Ello es así, porque la incorporación de nuevas tecnologías en los procesos productivos, ha modificado significativamente la estructura de los puestos de trabajo, así como los perfiles de conocimientos, habilidades y destrezas requeridos para desempeñarlos.

Algunos autores ${ }^{14}$ señalan que, como consecuencia de la difusión y aplicación de nuevas tecnologías, y de la organización empresarial flexible que de ella se deriva, tanto la estructura

\footnotetext{
${ }^{12}$ Sagasti R. Francisco, "Subdesarrollo, ciencia y tecnología. Una apreciación del rol de la universidad latinoamericana", en Patricio Dooner e Iván Lavados (compiladores), La Universidad Latinoamericana. Visión de una década. Santiago, CPU, 1984; Sunkel, Osvaldo. "La universidad latinoamericana ante el avance científico y técnico; algunas reflexiones", en Jorge A. Sábato (compilador), El pensamiento latinoamericano en la problemática cienciatecnología-desarrollo-dependencia, Buenos Aires, Ed. Paidós, 1975. pp.73-83. ${ }^{13}$ Brunner, José Joaquín, "La educación superior y la formación profesional en América Latina", en Revista Mexicana de Sociología, UNAM-IIS, Año LI/Núm. 3, julio-septiembre, 1989, p.247.

${ }^{14}$ Véase al respecto: Pérez, Carlota, "Nuevo patrón tecnológico y educación superior: Una aproximación desde la empresa", en Retos científicos y tecnológicos. No.3, Caracas, CRESALC/UNESCO, 1991, pp.23-49; Marchello, Joseph M., "Education for a technology age", en Futures, october, 1987, pp.555-565.
} 
académico-administrativa como el currículo de las universidades tenderán a modificarse de manera significativa. Así, a nivel institucional, se prevé el surgimiento de distintos tipos de universidades (internacionales, regionales y locales ${ }^{15}$ ), caracterizadas por modelos organizacionales descentralizados, participativos y flexibles, que superen la centralización, la jerarquía tradicional profesor-alumno y la organización de las disciplinas concebidas como compartimientos estancos.

A nivel curricular, los centros de educación superior tenderán a dar un mayor impulso al trabajo en equipo por encima del individual; al aprendizaje experimental; al desempeño de diversos papeles; a la interdisciplinariedad y a la utilización de nuevos métodos de expresión no cognitivos como medios para alentar la creatividad y la solución de problemas. Éstos y otros cambios que ya se vislumbran, implicarán también la adopción de nuevos métodos y procedimientos de evaluación de los estudiantes, según su contribución al logro de resultados.

De igual forma, en relación con el ejercicio y la práctica profesional, los egresados de las universidades se caracterizarán por asumir actitudes creativas e innovadoras, poseer hábitos de auto-aprendizaje, tener la capacidad de comunicarse con personas de otras disciplinas, trabajar más como consultores independientes que como empleados a tiempo completo en la industria o el Estado, asumiendo por lo tanto nuevos retos y liderazgos.

\section{CAMBIO TECNOLÓGICO Y EDUCACIÓN EN INGENIERÍA}

La experiencia histórica de diversos países, muestra que uno de los factores asociados a la explosión de nuevas tecnologías

${ }^{15}$ MaGinn, Noel. "Opciones para la educación superior en el momento en que América Latina ingresa a la economía mundial", en Revista Paraguaya de Sociología, Año 33, No.95, enero-abril, 1996, pp.7-24. 
es el incremento de profesionistas altamente calificados, especialmente en las áreas científicas y de ingeniería ${ }^{16}$. Ello se debe a que las nuevas tecnologías se basan esencialmente en el sofware y precisan un conjunto de habilidades y conocimientos sofisticados y más difíciles de obtener que mediante las técnicas de naturaleza mecánica. El cambio hacia el diseño de sistemas de producción en lugar de piezas separadas de maquinaria, supone conocimientos de ingeniería de sistemas que sólo se adquieren después de un entrenamiento de alto nivel y una práctica prolongada ${ }^{17}$.

El hecho de que las nuevas tecnologías -particularmente las tecnologías de la información- requieran complementar la destreza manual con facultades intelectuales sustentadas en saberes complejos y evolutivos, ha propiciado un importante

\footnotetext{
${ }^{16}$ Sobre este aspecto véanse: Boutzev, Cristo, "The engineer and the modern technological revolution", en Impact of Science and Society, Number 146. pp. 171-75; Milles, W., "Aspects of New Technology and its impacts on the Engineering Profesión", SPI International, Merlo Park, California, 1984; Telford, R. "Demography, Education and European Competitiveness". Proceedings of EIRMA Conference on Industrial R\&D and the Human Resource, London, U.K., 1992. pp. 12-23; Shleifer A., Murphy, M. and Vishny, R. "Industrial Policy, Call it what you will, the nations needs a plan to nurture growth", Business Week, April 6, 1992. pp.44-50. Para un análisis interesante del papel que jugaron los físicos e ingenieros de la Escuela Politécnica de la Universidad de Sao Paulo en la creación de la industria de las computadoras en Brasil véase: Langer D. Erick, "Generations of cientists and engineers: Origins of the Computer Industry in Brazil", en Latin American Research Review, Vol. XXV, No.2, 1989, pp.95-111.

${ }^{17}$ Cfr. Varios autores., Un horizonte sin certezas. América Latina ante la nueva revolución científico-técnica. Buenos Aires, Punto Sur Editores, 1987, p. 42.
} 
debate en torno a la enseñanza de la ingeniería y los nuevos perfiles de conocimientos, habilidades y destrezas que requerirán los ingenieros en el futuro ${ }^{18}$.

Este debate tiene su origen, por un lado, en el acelerado avance de los conocimientos científico-tecnológicos y, por el otro, en el creciente desfase que se observa entre la evolución tecnológica, las estructuras ocupacionales y los modelos de formación y práctica profesional de los ingenieros, los cuales -se plantea-deberán modificarse para asegurar el desarrollo tecnológico, el avance industrial y el bienestar social de los países.

Hoy, las críticas se centran en la incapacidad de la educación para formar ingenieros que participen tanto en el diseño como en la producción; en la formación de ingenieros más orientados a resolver problemas comunes en la industria que a estimular el cambio y la innovación tecnológicas.

En el ámbito educativo, el debate se centra entre el dominio de las ciencias básicas y las ciencias de la ingeniería,

${ }^{18}$ Para el caso inglés véase: Pollard, A. F., The Education and Training of Chartered Engineers for the 21st Century. The Fellowship of Engineering, London, February, 1989; Finneston M. Engineering our future. A report of inquiry into the engineering profession. HMSO Commnad No. 7794, London, January 1980; Archer, John, "Engineering Education en the 1990's". Para el caso francés: Angelino, Henri. "Situación de los ingenieros en Francia", Toulouse, 1993; para el caso norteamericano: Engineering Education and Practice in the United States. Foundations of our Techno-Economic Future. Committe on ther Education and Utilization of the Engineer, Comission on Engineering and Technical Systems, National Research Council. National Academy Press, Washington, D.C. 1985. Para un análisis del caso de México consúltense: Varios autores, "Ciencias Exactas e Ingeniería". Sexta Mesa Redonda del Seminario Sobre Educación Superior. México, El Colegio Nacional, 1979; Hanel, Jorge y Taborga Huascar. "Formación de los ingenieros frente a la globalización", en Revista de la Educación Superior. Vol.XX, No.2 (78), abril-junio, 1991. pp.37-45; Plata Olvera, Francisco, "El futuro de la enseñanza de la ingeniería", en Memoria del Primer Congreso Internacional de Problemática y Perspectivas de la Ingeniería Electromecánica. México, febrero 17-20, 1992. pp.90-93; Prospectiva de la formacion de ingenieros. México, SEFI, 1992; Casillas de León, Juan, "Evaluación de la educación superior y acreditación de ingeniería". Ponencia presentada en la XIX Conferencia Nacional de Ingeniería, ANFEI, junio, 1992. Girard, René, "Un ingeniero en el umbral del tercer milenio", en Técnica y Humanismo. Año XI, No.61, julio-agosto, 1991. 
y en la necesidad de introducir un mayor balance entre ciencia (análisis) y diseño (síntesis creativa) en el currículo de ingeniería.

Al nivel de la práctica y el ejercicio profesional, la discusión gira en torno a la educación para el trabajo y en el trabajo. Se ha constatado que una sólida preparación en matemáticas y en ciencias no bastan en sí mismas para proporcionar una adecuada formación de los ingenieros. La experiencia adquirida en la industria y el entrenamiento en el trabajo, amplían la perspectiva del ingeniero y lo familiarizan con los problemas productivos, proporcionándole habilidades para interactuar en grupo.

En cuanto a su posición y desempeño en la nueva organización industrial, los ingenieros se han desplazado cada vez más de la producción a la administración, requiriendo de un enfoque de ingeniería de producto, así como de conocimientos y técnicas específicos de gestión de personal, y de resolución de problemas y conflictos en grupos.

Debido a esta diversidad de formaciones, tareas y funciones requeridas por el avance tecnológico y por la nueva la estructura organizacional de las empresas, los países industrializados demandan hoy tres tipos de ingenieros: 1) aquellos que con una formación científica sólida, dotados de conocimientos en tecnología de computación, trabajen en problemas reales de diseño y de software; 2) ingenieros que participan en tareas importantes aunque rutinarias, utilizando -pero no desarrollando-software; y 3) "ingenieros funcionales", que participen en equipos de trabajo integrados por una variedad de individuos que colaboran en el desarrollo de un producto ${ }^{19}$.

${ }^{19}$ Cfr. Vargas Leyva, Ma. Ruth. "El estado actual de la formación de ingenieros. Criterios para la excelencia y la competitividad", en Revista de la Educación Superior, Vol.XXIV (2), No.94, abril-junio, 1995, pp.37-38. 


\section{ESBOZO DE LA FORMACIÓN DE INGENIEROS EN MÉXICO}

En México, la formación de profesionales en ingeniería no sólo es insuficiente en muchas áreas, sino que no parece corresponder a los nuevos modelos de organización del trabajo y de innovación tecnológica que se están introduciendo al país.

Un somero análisis de la evolución de la ingeniería mexicana manifiesta que, hasta principios de este siglo, los mecanismos de transferencia tecnológica más frecuente fueron la importación de ingenieros y técnicos, y la inversión extranjera directa.

Si bien a partir de los años veinte, México dispuso de un pequeño grupo de ingenieros -principalmente civiles, mecánicos y electricistas- que contribuyeron a la construcción de la infraestructura necesaria para el desarrollo agropecuario, industrial y los servicios, no fue sino hasta la década de los cuarenta cuando, con el crecimiento de la educación superior, el país pudo prescindir de ingenieros y técnicos extranjeros.

Durante los años cincuenta y principios de los sesenta, el gobierno destinó un importante monto de recursos para el establecimiento de un gran número de escuelas técnicas y profesionales dirigidas a la formación de técnicos medios e ingenieros ${ }^{20}$. Pese este impulso, el país empezó a experimentar un cierto déficit de este tipo de personal en distintas ramas industriales.

Hasta principios de los ochenta, la ingeniería mexicana había alcanzado un alto grado de autosuficiencia en algunas ramas (como la ingeniería civil, la ingeniería sísmica y la ingeniería hidráulica), pero en otras (como la ingeniería eléctrica y electrónica), la mayor parte de la tecnología debía importarse.

Por el lado de la educación, aunque el número de estudiantes matriculados en carreras de ingeniería continuó creciendo,

\footnotetext{
${ }^{20}$ Véase al respecto: Urquidi, Víctor y Lajous Vargas, Adrián. Educación superior, ciencia y tecnología en el desarrollo económico de México, México, El Colegio de México, 1969, cap.IV.
} 
su distribución por ramas o especialidades resultó inadecuado para el país. En efecto, un estudio de las tendencias seguidas por diferentes ramas de la ingeniería, entre 1930 y 1985, manifiesta tres cambios significativos: 1) una reducción de largo plazo de los campos clásicos de ingeniería (civil, mecánica, eléctrica, química y, sobre todo, agronómica y sanitaria); 2) un crecimiento moderado, de largo plazo (aunque con algunas variaciones) en especialidades más o menos tradicionales (ingeniería minera, hidráulica, topográfica y petrolera); 3) un acelerado crecimiento de nuevas especialidades, particularmente de la ingeniería industrial, electrónica y computación.

A pesar del elevado número de estudiantes, que cursaban éstas y otras carreras, la educación en ingeniería enfrentaba problemas tales como la falta de actualización de planes y programas de estudios, carencia de laboratorios adecuados, creciente subdivisión de carreras que dificultaban el ajuste entre oferta y demanda de trabajo, bajos índices de eficiencia terminal, falta de reconocimiento económico y social del trabajo de los ingenieros, etcétera.

En los últimos lustros, la matrícula de ingeniería siguió creciendo, aunque a un ritmo menor que en décadas anteriores. Según datos de la AMI, hasta 1992, México contaba con 292 planteles (entre universidades e institutos tecnológicos públicos y privados), que atendían a 270 mil estudiantes de ingeniería en 269 carreras.

Si bien el número de establecimientos que ofrecían estudios de ingeniería en todo el país no era despreciable, la proporción de ingenieros por cada mil habitantes -en comparación con las naciones desarrolladas- dejaba mucho que desear. Así, mientras que México disponía de 4 ingenieros por cada mil habitantes, Francia disponía de 6, Estados Unidos contaba con 12 y Japón con 19 ingenieros por cada mil habitantes.

De igual forma, a pesar de que el número de establecimientos que ofrecían estudios de ingeniería había crecido en todo el país, la mayoría de ellos se concentraba en sólo 12 
entidades (Distrito Federal, Coahuila, Puebla, Tamaulipas, Sonora, Chihuahua, Veracruz, Guanajuato, Nuevo León, Estado de México, Jalisco y Sinaloa), las cuales reunían al 60.2\% del total de las instituciones ${ }^{21}$.

Como resultado de esta concentración de instituciones en unas pocas entidades federativas, el $75 \%$ de los alumnos matriculados y el $70 \%$ de los egresados de alguna carrera de ingeniería, se ubicaban en sólo nueve entidades federativas (Distrito Federal, Nuevo León, Jalisco, Veracruz, Coahuila, Estado de México, Chihuahua, Tamaulipas y Puebla, en ese orden) ${ }^{22}$.

$\mathrm{Al}$ analizar el volumen de estudiantes matriculados en las carreras de mayor demanda, los datos de AMI muestran que éste permanecía fuertemente concentrado en unas pocas especialidades tradicionales y modernas. En consecuencia, de los 270 mil estudiantes inscritos en carreras de ingeniería en 1992, el 95.8\% se concentraba en las especialidades de sistemas (19.4\%); mecánica y eléctrica (19.4\%); industrial (19.2\%); comunicaciones y electrónica (14.1\%); ingeniería civil (13.7\%); y química $(10.1 \%)^{23}$.

Por lo que toca a los alumnos de primer ingreso en carreras de ingeniería, se observaba que si bien éstos aumentaban de manera lenta, existían algunas carreras en las cuales se habían incrementando considerablemente (tales como ingeniería en computación y sistemas, electrónica, industrial y mecánica), mientras que en otras se reducían de manera drástica (particularmente en ingeniería agronómica, geológica, pesquera, topográfica e hidráulica) ${ }^{24}$.

Los datos anteriores sugieren que aun cuando a principios de los noventa, la matrícula de ingeniería continuaba creciendo en forma significativa, la misma seguía concentrada en algunas

\footnotetext{
${ }^{21}$ AMI, Estado del Arte $\ldots$, p. 128.

${ }^{22}$ Ibid. p.133.

${ }^{23}$ Ávila Galinzoga, Jésus, op. cit., pp.10-11.

${ }^{24}$ AMI, Estado del Arte..., p. 131.
} 
entidades federativas y en unas pocas carreras de corte tradicional y moderno, en tanto que era muy reducida la población escolar inscrita en otras que resultaban indispensables para acelerar el desarrollo agropecuario, pesquero e industrial del país.

En relación con la eficiencia terminal en las carreras de ingeniería que imparten las instituciones de educación superior públicas, estudios ${ }^{25}$ realizados a fines de los años ochenta indican que ésta era, en promedio, del 40\%, y que los estudiantes de algunas de estas instituciones, como la UNAM, requerían cerca de ocho años -desde la primera inscripción- para obtener su título.

Por lo que se refiere a los estudios de posgrado en ingeniería, el diagnóstico de AMI ${ }^{26}$ señala que hasta 1992 se tenían registradas 222 maestrías en ingeniería en todo el país, con una población de casi 5500 estudiantes, mientras que los programas de doctorado ascendían a 24 , con una matrícula de 222 estudiantes. Entre las especialidades de maestría que absorbían el mayor número de estudiantes destacaban: ingeniería en sistemas (20.3\%); agronómica (11.6\%); mecánica y eléctrica (11.1\%); industrial (9\%) y química (8.3\%). De las especialidades de doctorado, el mayor porcentaje de estudiantes se encontraba en las especialidades de ingeniería agronómica (28.1\%); civil (21.5); mecánica y eléctrica (19.3) y química (5.3\%). Adicionalmente, como en el caso de la licenciatura, cerca $60 \%$ de los estudiantes matriculados en algún programa de maestría en ingeniería y el 50\% de los inscritos en alguno de doctorado, se concentraban en sólo dos entidades federativas (el Distrito Federal y Nuevo León).

De lo anterior se desprende que, a pesar del incremento de estudiantes de posgrado en ingeniería, su número continuaba siendo sumamente bajo para satisfacer los requerimientos de

${ }^{25}$ Entrevista a Daniel Reséndiz Núñez titulada: "La ingeniería y los retos del futuro", publicada en Ingeniería Civil, No.261, mayo de 1989, p.23.

${ }^{26}$ Galinzoga Ávila, Jesús, op.cit., pp.12-14. 
investigación e innovación tecnológica de la industria, expuesta ya a una intensa competencia internacional.

Finalmente, en lo que se refiere a la situación de los ingenieros en el mercado de trabajo, una encuesta realizada por la propia AMI sobre una muestra de 2485 casos destacaba que, en cuanto al nivel jerárquico que ocupaban los ingenieros dentro de su organización, el 50\% de los encuestados se situaba en puestos de tercer nivel, el $22 \%$ se ubicaba en puestos de segundo nivel y el $23 \%$ se desempeñaba como empleados.

Tomando en cuenta el área específica de trabajo (excluyendo a los académicos de tiempo completo en las instituciones de educación superior), los datos obtenidos indicaban que un $25 \%$ de los ingenieros encuestados ocupaba algún puesto de administración o finanzas, superando con mucho otras áreas tradicionales de trabajo de los ingenieros, tales como: diseño y cálculo (19\%); manufactura (16\%); investigación y desarrollo (10\%); mantenimiento (9\%); ventas o mercadotecnia (9\%), y otras (12\%).

\section{¿CONVERGENCIA O DIFERENCIACIÓN EN LA ENSEÑANZA DE LA INGENIERÍA?}

Como puede observarse, si bien México disponía a principios de los años 90 de un número adecuado de estudiantes e instituciones dedicadas a la enseñanza de la ingeniería, una proporción importante de la matrícula seguía concentrada en algunas entidades federativas y en unas pocas carreras de corte tradicional y moderno, en tanto que era muy reducida la población escolar inscrita en otras carreras igualmente indispensables para el desarrollo del país.

Un factor adicional, que desde hace varias décadas ha influido tanto en la diferenciación institucional como en la heterogeneidad de la formación universitaria, ha sido la tendencia cada vez mayor a la internacionalización de la educación superior en general y de la enseñanza de la ingeniería 
en particular. Aunque en el caso de México, la tendencia a la internacionalización (o convergencia) de la educación superior no es algo nuevo, algunos análisis recientes ${ }^{27}$ sugieren que ésta se ha ido profundizando como consecuencia de la firma del Acuerdo Trilateral de Libre Comercio (TLC) con Estados Unidos y Canadá.

En efecto, a principio de 1993, motivados por la firma del Acuerdo de Libre Comercio con Estados Unidos y Canadá, varios colegios y asociaciones profesionales de ingenieros ${ }^{28}$ crearon el Comité Mexicano para la Práctica Internacional de la Ingeniería (COMPII), con el fin de representar a los colegios de ingenieros mexicanos frente a sus similares de Canadá y Estados Unidos -el Canadian Council of Professional Engineering (CCPE) y el United States Council for International Engineering Practice (USCIEP), respectivamente-, en aquellos aspectos relacionados con el otorgamiento mutuo de licencias para la prestación de servicios profesionales en distintas especialidades de la ingeniería, en el marco del TLCAN. Sin embargo, puesto que México no dispone de un sistema de acreditación de títulos y grados basado en la constatación por quienes ejercen la profesión (es decir, a través de colegios o agrupaciones profesionales), como sucede en Canadá y los

\footnotetext{
27 Para una discusión más amplia de estos aspectos véase: Méndez Lugo, Bernardo, "Homologación, certificación y acreditación en el contexto del TLC: asimetrías nacionales y vulnerabilidad del profesional mexicano", en Educación superior y sociedad, Vol.6, No.2, 1995, pp.181-99; Castaños, Heriberta, Didriksson, Axel y Newson, Janice, "Reshaping the educational agendas of mexican universities", en Jean Currie y Janice Newson (editores), Universities and globalization. Critical perspectives, SAGE Publications, USA, 1998, pp.275-93; Marúm Espinosa, Elia, "Las profesiones y la educación superior en el marco de los procesos de integración económica de América Latina", en Revista de la Educación, SAGE Publications, USA, 1998, pp. 275-93; Marúm Espinosa, Elia, "Las profesiones y la educación superior en el marco de los procesos de integración económica de América Latina”, en Revista de la Educación Superior, Vol.XXVIII (2), No.110, abril-junio, 1999, pp.21-39. ${ }^{28}$ El Colegio de Ingenieros Mecánicos Electricistas (CIME); el Colegio de Ingenieros Químicos y Químicos, el Colegio de Ingenieros Civiles de México, y la Federación de Colegios de Ingenieros Civiles.
} 
Estados Unidos, la Asociación Nacional de Escuelas y Facultades de Ingeniería (ANFEI) propuso, en ese mismo año (1993), la creación del Sistema Mexicano de Acreditación de Programas de Ingeniería, cuyos criterios y prácticas de operación deberían ser formulados por los CIEES, y por los colegios y asociaciones profesionales de ingeniería. Como resultado de ello, en 1994 se formó el Consejo de Acreditación de la Enseñanza de la Ingeniería A.C. (CACEI), cuyos fines son: contribuir al mejoramiento de la calidad de la enseñanza de la ingeniería en las instituciones públicas y privadas; coadyuvar al establecimiento de programas y modelos de enseñanza de la ingeniería acordes con los avances de la ciencia; contribuir al mejoramiento de la calidad del ejercicio profesional de la ingeniería y, sobre todo, llevar a cabo los procesos de acreditación de programas educativos de ingeniería ${ }^{29}$. Entre los aspectos que este organismo toma en cuenta para realizar la acreditación ${ }^{30}$, destacan: las características de los programas académicos, el personal docente, los alumnos, el plan de estudios, los métodos de enseñanza-aprendizaje, la infraestructura, la investigación y los recursos financieros.

Muy ligado al aspecto de la acreditación, también en 1994, los organismos representativos de las asociaciones de ingenieros de los tres países firmantes del TLCAN (el COMPII mexicano, el CCPE canadiense y el USCIEP norteamericano), suscribieron una declaración de intención ${ }^{31}$ para facilitar la

\footnotetext{
${ }^{29}$ Cfr. Ocampo Canabal, Fernando, "La acreditación y su relación con la calidad de la enseñanza de la ingeniería", en Memoria de la Reunión Nacional sobre Regulación de las Profesiones. Situación Actual y Prospectiva, México, SESIC/DGP/SEP, noviembre, 1995, pp.33-34.

${ }^{30} \mathrm{La}$ acreditación es un reconocimiento explícito que el CACEI hace público por los medios que considere convenientes, de que el programa de la carrera de ingeniería evaluado cumple con los requisitos esenciales de calidad establecidos conforme a la experiencia de evaluación educativa tanto en México como en otros países.

${ }^{31}$ Véase "Convenio de intercambio de servicios profesionales dentro del TLC" en el cual se alude a la Declaración Interna de Reconocimiento Mutuo de Ingenieros, en Ingeniería Civil, No.303, julio, 1994, p.47.
} 
movilidad para el libre tránsito de los ingenieros en el territorio de los tres países. Como resultado de dicho acuerdo, se estableció un procedimiento para el otorgamiento de licencias temporales para ingenieros que pretenden ejercer la práctica de la ingeniería en el territorio de cada país. Este procedimiento contempla un esquema general aplicable a los tres países y considera dos opciones. La primera, en el caso de los ingenieros que tienen licencia para ejercer en su país y son graduados de un programa acreditado, implica revisar su formación en ingeniería, demostrar doce años de experiencia profesional y tener conocimiento del idioma y de las leyes locales del país en donde se desea ejercer la práctica de la ingeniería. En la segunda alternativa, para el caso de los ingenieros no egresados de un programa acreditado, se revisa su formación, se les exigen dieciséis años de experiencia profesional, y conocer el idioma y las leyes del país en que se desea ejercer ${ }^{32}$.

Hasta 1997, la ingeniería era la primera y única profesión que, bajo el TLCAN, había aprobado un acuerdo de mutuo reconocimiento con sus contrapartes canadiense y norteamericana. Según J. Mallea ${ }^{33}$, el Acuerdo estableció criterios para el mutuo reconocimiento que: 1) están basados en criterios objetivos y transparentes, tales como la competencia y la capacidad para proporcionar un servicio; 2) no contempla más cargas que las necesarias para asegurar la calidad de un servicio; y 3) no constituye una restricción encubierta para el suministro internacional de un servicio.

Aun cuando el TLCAN -firmado entre Canadá, Estados Unidos y México- alentó las expectativas de un acuerdo para la movilidad de la ingeniería entre los tres países, después de varios años de negociaciones, el acuerdo para la práctica

\footnotetext{
32 "Ingeniería y TLC", entrevista con el ingeniero Fernando Favela Lozoya, Coordinador del Comité Mexicano para la Práctica Internacional de la Ingeniería, en Ingeniería Civil, No.309, enero, 1995, pp.20-21.

${ }^{33}$ Mallea, John R., International trade in professional and educational service:implications for the professions and higher education, Paris, OECD, Centre for Educational Reseach and Innovation, 1998, p.6.
} 
abierta de la ingeniería a través de as fronteras de los tres países no ha podido llevarse a la práctica ${ }^{34}$.

Como puede observarse, si bien el TLC está propiciando un mayor intercambio de bienes, de conocimientos científicotecnológicos y de recursos humanos calificados entre las tres naciones, diversos estudios sugieren también que tal situación podría conducir -vía los procesos de acreditación institucional y estandarización curricular- al alejamiento de muchas profesiones (particularmente de la ingeniería) de las necesidades económicas y sociales del país ${ }^{35}$.

En este contexto, el reto que se plantea a las instituciones de educación superior en general y a las escuelas de ingeniería en particular, es cómo, bajo qué condiciones y a través de qué mecanismos, insertarse en la dinámica de los procesos de internacionalización y convergencia de los sistemas educativos y de la enseñanza de la ingeniería a nivel mundial, sin abandonar la tradición histórica, los valores y los fines que han orientado el quehacer de las escuelas y facultades de ingeniería mexicanas de carácter público.

Cabe señalar que la internacionalización o "convergencia" -como algunos autores han denominado también a este proceso- de los sistemas de educación superior no implica que todos se conviertan en uno solo, sino que sean gobernados cada vez más por procedimientos, pautas organizacionales y curricula similares, impuestos por la dinámica del cambio tecnológico y la globalización de la economía ${ }^{36}$.

${ }^{34}$ Jones, C., Russel, "Developments in engineering education and accreditation in the United States", World Expertise LLC Falls Church, VA, USA, en www.sefi/dk/papers/pdf/23pdf

35 Para una discusión más amplia de este aspecto véase: Méndez Lugo, Bernardo, "Homologación, certificación y acreditación en el contexto del TLC: asimetrías nacionales y vunerabilidad del profesional mexicano", en Educación Superior y Sociedad, Vol.6, No.2, 1995, pp.181-199.

${ }^{36}$ Schugurensky, Daniel, "La reestructuración de la educación superior en la era de la globalización: ¿Hacia un modelo heterónomo?», en Armando Alcántara, Ricardo Pozas y Carlos Torres, Educación, democracia y desarrollo en el fin de siglo, México, Ed. Siglo XXI, 1998, p.124. 
En la actualidad es posible distinguir dos principales modelos o paradigmas de convergencia de la educación superior a nivel internacional: el paradigma competitivo, característico de las instituciones anglosajonas, y el paradigma cooperativo de las instituciones de educación superior de Europa Continental. Aunque cada uno de estos modelos tiene raíces históricas diferentes, también observan importantes coincidencias que se manifiestan en una menor intervención Estatal, y en contraste, una mayor participación del sector privado; mayor desregulación institucional y diversificación curricular, una tendencia hacia la descentralización administrativa, mayor competencia por estudiantes y recursos, y mayor asociación entre universidades y empresas.

En México, algunas de estas tendencias se han venido manifestando en los últimos lustros en diversas escuelas y facultades de ingeniería del país, las cuales han adoptado formas de organización académico-administrativa más descentralizadas (de tipo departamental), han diversificado su oferta de carreras y/o actualizado las existentes atendiendo a los avances tecnológicos; han flexilibilizado su curricula, y modernizado los métodos de enseñanza aprendizaje (sobre todo utilizando la computadora, el CD Room y el Internet). De igual forma, siguiendo las tendencias internacionales, han enfatizado el conocimiento de idiomas vía el intercambio de estudiantes, o bien, a través de estancias de estudio o trabajo en el extranjero, y se han acercado cada vez más a las empresas a través de la venta de servicios, investigación y consultoría. Por último, como consecuencia de las políticas de evaluación de la educación superior, se han creado nuevos organismos de evaluación institucional, de pares, así como exámenes de calidad profesional para algunas carreras de ingeniería.

A pesar de éstas y otras reformas experimentadas por la educación en ingeniería en México, las mismas no pueden ser consideradas como signos inequívocos de convergencia y, por tanto, de homogenización y estandarización, observándose incluso movimientos en contrario que -como la diversificación de carreras, la 
diferenciación institucional y la acreditación programáticaparecen dar pie a procesos de diferenciación, y, consecuentemente, a un mayor acercamiento de ella con el mercado.

Se ha señalado que si el país desea consolidar la nueva estrategia de desarrollo y aumentar la productividad y la competitividad de la industria nacional, los aproximadamente 360 mil ingenieros de que disponemos ${ }^{37}$, deberán multiplicarse por 20 en los próximos veinte años. Dicho requerimiento puede satisfacerse si se mantienen las tendencias actuales de crecimiento de la matrícula y se incrementa la eficiencia terminal por arriba del $35 \%$ actual ${ }^{38}$. Con todo, si estas metas no van acompañadas de un cambio en las características de la formación profesional que vaya más allá de las demandas del mercado, el sólo aumento de la cantidad de egresados no contribuirá a elevar los niveles de productividad y competitividad de la industria nacional ni a reducir la dependencia tecnológica del país. Para ello, los ingenieros mexicanos del nuevo milenio no sólo deberán estar mejor formados en ciencias básicas, ingeniería y tecnología, sino que deberán atender más los requerimientos económicos, sociales y tecnológicos del país en la actual etapa de transición. Deberán, sobre todo, interrogarse acerca de qué producir, para quién y con qué fines últimos. Sólo a partir de éstos y otros cuestionamientos, estarán en condiciones de redefinir su papel como grupo profesional, y contribuir profesional, ética y socialmente -como lo hicieron sus antecesores- a impulsar nuevamente el crecimiento económico y el bienestar de México.

\section{CONCLUSIONES}

La inserción de nuestro país al llamado bloque de América del Norte ha propiciado, en la última década, transformaciones

${ }^{37}$ Fernández Zayas, José Luis, "La ingeniería mexicana. Reflexiones acerca de su situación actual", en Arturo Menchaca (coordinador), Las ciencias exactas en México, FCE/CONACULTA, 2000, p.240.

${ }^{38}$ Reséndiz Núñez, Daniel y Elizondo, Jorge. op.cit., p.87. 
importantes en todos los ámbitos, especialmente en la enseñanza superior. Como resultado de las políticas instrumentadas en este nivel, las instituciones de enseñanza superior se han visto presionadas para elevar la calidad de la enseñanza, reformar su estructura curricular, actualizar sus procesos de enseñanza aprendizaje, estimular la superación del personal académico y aceptar nuevas reglas de financiamiento basadas en las mejoras académicas y organizacionales que logren llevar a cabo, el establecimiento de procesos de evaluación institucional y de acreditación y reconocimiento de estudios con instituciones nacionales y extranjeras.

En el ámbito del ejercicio profesional de la ingeniería, uno de los efectos más importantes de la firma del TLCAN, ha sido la posible apertura del mercado de trabajo para los ingenieros de los tres países signatarios. Desde 1991 de manera informal, y a partir de 1994 más formalmente, las asociaciones profesionales de los tres países -representadas por el CCPE canadiense, la USCIEP norteamericana y el COMPII mexicanohan venido sosteniendo una serie de reuniones a fin de establecer criterios y procedimientos para el reconocimiento mutuo de licencias para la prestación de servicios profesionales en distintas especialidades, en el marco del TLCAN.

A pesar de estos esfuerzos, hasta 1997 sólo el Estado de Texas, en los Estados Unidos, y varias provincias canadienses habían mostrado interés por concretar el Acuerdo de Reconocimiento Mutuo relativo a la prestación de servicios profesionales. En la actualidad, México define los procedimientos y mecanismos que aplicará para la expedición de licencias temporales, las cuales resultan indispensables para asegurar la reciprocidad en el ingreso de los ingenieros con los otros dos países firmantes del TLCAN. Tales procedimientos y mecanismos deberán, sin embargo, considerar las asimetrías y disparidades existentes entre los tres países, y evitar la competencia desleal para los profesionistas mexicanos. 
ANGELINO, Henri., Situación de los ingenieros en Francia., Toulouse., 1993 (fotocopia).

ARCHER, John., Engineering Education in the 1990's.

ÁVILA GALINZOGA, Jesús., "Enseñanza de la ingeniería en México", en Memoria del II Congreso Internacional. El estado del arte y prospectiva de la ingeniería en México y el mundo., Tomo I., México., Academia Mexicana de Ingeniería/ CONACYT., 1993., pp.437-513.

BENACHENHOU, Abdellatyff, "Adquisición de conocimientos en los países subdesarrollados. Situación actual y perspectivas"., en Foro Internacional., Vol.XXVIII., No.2., octubre-diciembre., 1987.

BOUTZEV, Cristo., "The engineer and the modern technological revolution", en Impact of Science and Society., No.146., pp.171-175.

BRUNNER, José Joaquín., "La educación superior y la formación profesional en América Latina"., en Revista Mexicana de Sociología., UNAM-IIS., Año LI/No.3., julio-septiembre., 1989., p.247.

BRUNNER, José Joaquín., Recursos humanos para la investigación científica en América Latina., Santiago., FLACSO/IDRC., 1989.

CASILLAS DE LEÓN, Juan., "Evaluación de la educación superior y acreditación de ingeniería", ponencia presentada en la XIX Conferencia Nacional de Ingenieria., ANFEI., junio., 1992. CASTAÑOS, Heriberta; DIDRIKSSON, Axel y NEWSON, Janice., "Reshaping the educational agendas of mexican universities"., en Jean Curie y Janice Newson (edit.)., Universities and globalization. Critical perspectives., SAGE Publications., USA., 1998., pp.275-293.

CASTELlS, Manuel; BARRERA, Antonio, et.al., El desafío tecnológico en España. España y las nuevas tecnologías., Madrid., Alianza Editorial., 1986. 
CEPAL., Transformación productiva con equidad., Santiago de Chile., UNESCO., marzo., 1990.

CEPAL., Educación y conocimiento: eje de la transformación productiva con equidad., Santiago de Chile., UNESCO., marzo., 1992.

CIMOLI, Mario y DOSI, Giovanni., "Tecnología y desarrollo. Algunas consideraciones sobre los recientes avances en la economía de la innovación", en Mikel Gómez; Miguel Sánchez y Enrique de la Puerta (comps.)., El cambio tecnológico hacia el nuevo milenio., Barcelona., Ed. Icaria., 1992.

"Declaración interna de reconocimiento mutuo de ingenieros"., en Ingeniería Civil., No. 309., enero., 1995., pp.20-21.

Engineering Education and Pratice in the United States. Foundations of our Techno-Economic Future., Comitte on ther Education and Utilization of the Engineer Comisión on Engineering and Thecnical Systems., National Research Council. National Academy Press., Washington, D.C., 1985.

Entrevista a Daniel Reséndiz Núñez titulada: «La ingeniería y los retos del futuro"., en Ingeniería Civil., No.261., mayo., 1989., p.23.

FANJZYLBER, Fernando., "Reflexiones sobre ciencia, tecnología y sociedad", en GONZÁLEZ CASANOVA, Pablo y AGUILAR CAMÍN, Héctor., México ante la crisis., México., Siglo XXI., 1986.

SEGAL, Aarón., "De la transferencia de tecnología a la institucionalización de la ciencia y la tecnología"., en Revista de Comercio Exterior., Vol.37., No.12., diciembre., 1987., pp.983-993. FERNÁNDEZ ZAYAS, José Luis, “La ingeniería mexicana. Reflexiones acerca de su situación actual", en Arturo Menchaca (coordinador), Las ciencias exactas en México, FCE/CONACULTA, 2000, p.237-247.

FINNESTON, M., Engineering our future. A report of inquiry into the engineering profession., HMSO., Commnad No. 7794., London., January., 1980.

GILL-CHIN, Limm., "La transformación dinámica y el papel del gobierno: la lección de Corea y de los países de industrialización 
reciente", en Omar Martínez Legorreta (comp.)., Industria, comercio y Estado. Algunas experiencias de la Cuenca del Pacífico., México., El Colegio de México., 1991.

TUN-JEN, Chen., "Características distintivas del desarrollo económico de Taiwán, con referencia particular al papel de las medianas y pequeñas empresas"., en Omar Martínez Legorreta (comp.)., Industria, comercio y Estado. Algunas experiencias de la Cuenca del Pacífico., México., El Colegio de México., 1991. GIRARD, René., "Un ingeniero en el umbral del tercer milenio"., en Técnica y Humanismo., Año XI., No.61., julio-agosto., 1991. HANEL, Jorge y TABORGA HUASCAR. «Formación de los ingenieros frente a la globalización"., en Revista de Educación Superior., Vol.XX., No. 2 (78)., abril-junio., 1991., pp.37-45. "Ingeniería y TLC", entrevista con el ingeniero Fernando Favela Lozoya, Coordinador del Comité Mexicano para la Práctica Internacional de la Ingeniería, en Ingeniería Civil, No.309, enero, 1995, pp.20-24.

JONES C., Russel., "Developments in engineering education and accreditation in the United States"., World Expertise LLC Falls Church., VA., USA., en www.sefi/dk/papers/pdf/23pdf LOREY, E. David., "The developmente of engineering expertise for Social and Economic Modernization in México sinde 1929", en James Wilkie (editor)., Society and Economy in México., UCLA., Los angeles., 1990., pp.71-102.

MAGINN, Noel. "Opciones para la educación superior en el momento en que América Latina ingresa a la economía mundial", en Revista Paraguaya de Sociología, Año 33, No.95, enero-abril, 1996, pp.7-24.

MALLEA, John R., International trade in profesional and educational service: implications for the professions and higher education., París., OECD., Centre of Educational Research and Innovation., 1998.

MARCHELLO, Joseph M., "Education for a technology age»., en Futures., octubre., 1987., pp.555-565. 
MARÚM ESPINOSA, Elia., "Las profesiones y la educación superior en el marco de los procesos de integración económica de América Latina"., en Revista de la Educación Superior., Vol.XXVIII (2)., No. 110., abril-junio., 1999., pp.21-39.

MÉNDEZ LUGO, Bernardo., "Homologación, certificación y acreditación en el contexto del TLC: asimetrías nacionales y vulnerabilidad del profesional mexicano"., en Educación Superior y Sociedad., Vol.6., No.,2., 1995., pp.181-199.

MILLES, W., Aspects of New Technology and its Impacts on the Engineering Profesión., SPI International., Merlo Park., California., 1984.

OCAMPO CANABAL, Fernando., "La acreditación y su relación con la calidad de la enseñanza de la ingeniería", en Memoria de la Reunión Nacional sobre Regulación de las Profesiones. Situación Actual y Prospectiva., México SESIC/DGP/SEP., noviembre., 1995., pp.34-35.

PÉREZ, Carlota., "Las nuevas tecnologías, una visión de conjunto"., en Carlos Ominami (ed.)., La tercera revolución industrial. Impactos internacionales del actual viraje tecnológico., Buenos Aires., Grupo Editor Latinoamericano., 1986., 43-89.

PÉREZ, Carlota., "Nuevo patrón tecnológico y educación superior: una aproximación a la empresa"., en varios autores., Reunión internacional de reflexión sobre nuevos roles de la educación superior a nivel mundial: el caso de América Latina y del Caribe, futuro y escenarios deseables., Vol.3., Retos científicos y tecnológicos., Caracas., UNESCO/CRESALC, 1991., pp.23-49.

PLATA OLVERA, Francisco., "El futuro de la enseñanza de la ingeniería", en Memoria del Primer Congreso Internacional de Problemática y Perspectiva de la Ingeniería Electromecánica., México., febrero 17-20., 1992., pp.90-93.

POLLARD., A.F., The Education and Training of Chartered Engineers for the 21st. Century., The Fellowship of Engineering, London, February., 1989. 
RUÍZ DURÁN, Clemente., "El bienestar social, el desarrollo económico y la organización empresarial"., todos estos trabajos se encuentran en Omar Martínez Legorreta (comp.)., Industria, comercio y Estado. Algunas experiencias de la Cuenca del Pacífico., México., El Colegio de México., 1991. SAGASTI, R. Francisco., "Ciencia y tecnología y la encrucijada de América Latina", en Manuel Gamella y Mar Hernández de Felipe (coords.)., Nuevas tecnologías y orden económico internacional., Madrid., FUNDESCO., 1990.

SCHUGURENSKY, Daniel., "La reestructuración de la educación superior en la era de la globalización: ¿Hacia un modelo heterónomo?»., en Armando Alcántara, Ricardo Pozas y Carlos Torres., Educación, democracia y desarrollo en el fin del siglo., México., Ed. Siglo XXI., 1998., p.124.

SEFI., Prospectiva de la formación de ingenieros., México., Tomo I., 1992.

SEGAL, Aaron, "De la transferencia de tecnología a la institucionalización de la ciencia y la tecnología", en Revista Comercio Exterior, Vol. 37, No.12, diciembre de 1987, pp.983-993.

SHLEIFER A., Murphy and VISHNY, R., "Industrial Policy, Call it what you will, the nations need a plan to nurture growth", Business Week., 1992., pp.44-50.

SUNKEL, Osvaldo., "La universidad latinoamericana ante el avance científico y técnico; algunas reflexiones"., en Jorge A. Sábato (comp.)., El pensamiento latinoamericano en la problemática ciencia-tecnología-desarrollo-dependencia., Buenos Aires., Ed. Paidós., 1975., pp.73-83.

TEITEL, Simón y WETSPHAL, Larry E., Cambio tecnológico y desarrollo industrial., México., FCE., 1990.

TELFORD, R., "Demography, Education and European Copetitiveness"., Proceedings of EIRMA Conference on Industrial R\&D and the Human Reosurce., London., UK., 1992., pp.12-23. 
URQUIDI., Víctor y LAJOUS VARGAS, Adrián., Educación superior, ciencia y tecnología en el desarrollo económico de México., México., El Colegio de México., 1969.

VARGAS LEYVA, María Ruth., "El estado actual de la formación de ingenieros. Criterios para la excelencia y la competitividad"., en Revista de la Educación Superior., Vol.XXIV (2)., No. 94., abril-junio., 1995., pp.37-38.

Varios autores. "Ciencias Exactas e Ingeniería". Sexta Mesa Redonda del Seminario sobre Educación Superior., México., El Colegio Nacional., 1979.

Varios autores., Un horizonte sin certezas. América Latina ante la nueva revolución científico-técnica., Buenos Aires., Punto Sur Editores., 1987.

Varios autores., "Situación de la ingeniería en México", en Estado del arte de la ingeniería en México y en el mundo., Tomo II., México., Academia Mexicana de Ingeniería (AMI)/CONACYT., mayo., 1993. 